\title{
Bardoxolone conjugation enables targeted protein degradation of BRD4
}

\author{
Bingqi Tong ${ }^{1,2,5}$, Mai Luo ${ }^{1,2,5}$, Yi Xie $^{1,2}$, Jessica N. Spradlin ${ }^{1,2}$, John A. Tallarico ${ }^{2,3}$, \\ Jeffrey M. McKenna ${ }^{2,3}$, Markus Schirle ${ }^{2,3}$, Thomas J. Maimone ${ }^{1,2 \bowtie}$ \& Daniel K. Nomura ${ }^{1,2,4} \bowtie$ \\ Targeted protein degradation (TPD) has emerged as a powerful tool in drug discovery for the \\ perturbation of protein levels using heterobifunctional small molecules. E3 ligase recruiters remain \\ central to this process yet relatively few have been identified relative to the $\sim 600$ predicted human E3 \\ ligases. While, initial recruiters have utilized non-covalent chemistry for protein binding, very recently \\ covalent engagement to novel E3's has proven fruitful in TPD application. Herein we demonstrate \\ efficient proteasome-mediated degradation of BRD4 by a bifunctional small molecule linking the \\ KEAP1-Nrf2 activator bardoxolone to a BRD4 inhibitor JQ1.
}

Targeted protein degradation (TPD) has emerged as a powerful therapeutic modality for drug discovery ${ }^{1-11}$. One strategy available to achieve this therapeutic modality employs heterobifunctional small-molecules known as degraders or proteolysis-targeting chimeras (PROTACs) that are comprised of three constitutive components: a E3 ligase recruiter; a linker; and a ligand to target a protein of interest (POI). By recruiting the E3 ligase to the POI, the resultant PROTAC is able to induce ubiquitination and degradation of the POI in a proteasomedependent manner (Fig. 1A $)^{1-11}$. While this therapeutic modality has tremendous potential, a major challenge overshadowing the area is that there are only a small number of E3 ligase recruiters that have been identified, this despite there being in excess of 600 predicted E3 ligases. Known and popularized E3 ligase recruiters include thalidomide-type immunomodulatory drugs (IMiDs) that recruit cereblon (CRBN), hydroxyprolinebased ligands for the von-Hippel Lindau (VHL) E3 ligase, nutlins that bind to MDM2, and ligands against cIAP (Fig. 1B $)^{1-15}$. While these recruiters bind reversibly to their corresponding E3 ligases, recent studies have revealed that reactive small-molecules that can covalently target E3 ligases can also be used as E3 ligase recruiting modules to potently degrade target proteins in TPD applications. These electrophilic moieties include derivatives of the terpene natural product nimbolide that covalently binds to a disordered cysteine on the E3 ligase RNF114, CCW 16 that reacts with a zinc-coordinating cysteine on the E3 ligase RNF4, and KB02 that covalently targets the cullin E3 ligase DCAF16 (Fig. 1B) ${ }^{16-19}$.

Based on the success of covalent E3 ligase recruiters, which have the potential to exploit the vast array of nucleophilic amino acid residues within proteins, we postulated that covalent and reversible E3 ligase recruitment could be a third, underexplored area in PROTAC development (Fig. 1B). As a possible mechanism of action, reversible covalent modification offers the potential for sustained target engagement, while avoiding permanent protein modification - a feature of particular interest given the catalytic nature of PROTACs. While this concept has proven powerful in drug discovery settings $\mathrm{s}^{20-25}$, we are unaware of its successful employment in E3 ligase recruitment ${ }^{26,27}$. Herein we investigate the small molecule bardoxolone, which possesses a highly reactive-yet reversible- $a$-cyanoenone hetero Michael acceptor, as the basis for the first protein degrader exploring this concept.

\footnotetext{
${ }^{1}$ Department of Chemistry, University of California, Berkeley, CA 94720, USA. ${ }^{2}$ Novartis-Berkeley Center for Proteomics and Chemistry Technologies, Berkeley, CA, USA. ${ }^{3}$ Novartis Institutes for BioMedical Research, Cambridge, MA 02139, USA. "Departments of Molecular and Cell Biology and Nutritional Sciences and Toxicology, University of California, Berkeley, CA 94720, USA. ${ }^{5}$ These authors contributed equally: Bingqi Tong and Mai Luo. ${ }^{\square}$ email: maimone@berkeley.edu; dnomura@berkeley.edu
} 
A

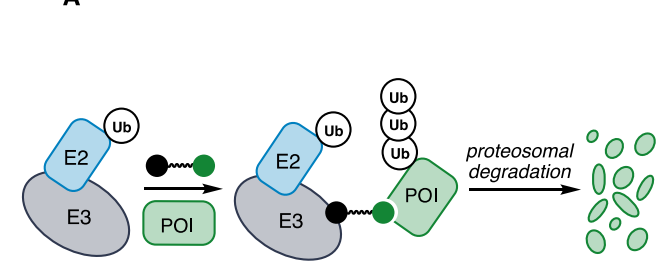

B

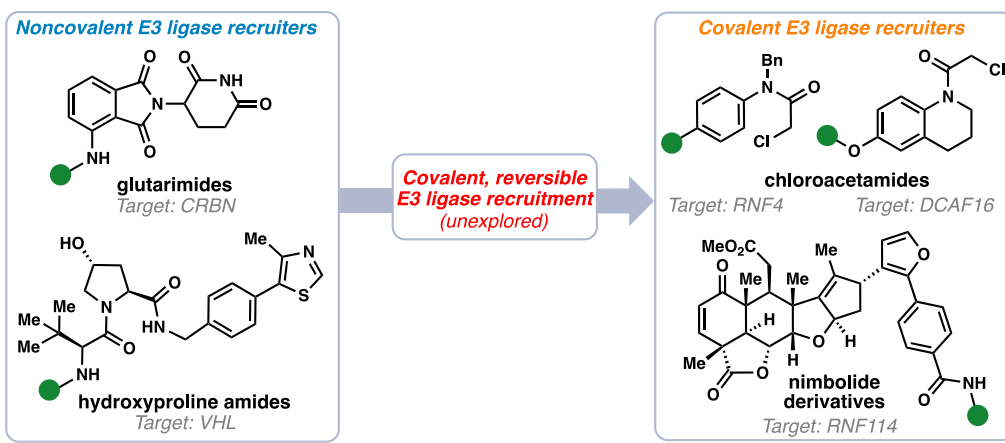

Figure 1. (A) Targeted protein degradation using bifunctional small molecules. (B) Selected examples of E3 ligase recruiters of varying degrees of covalent engagement.

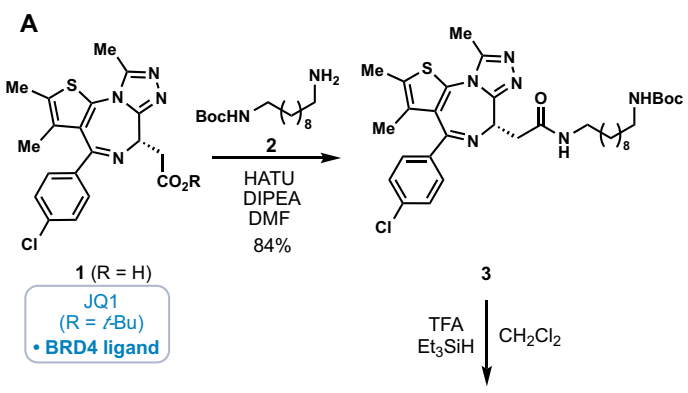

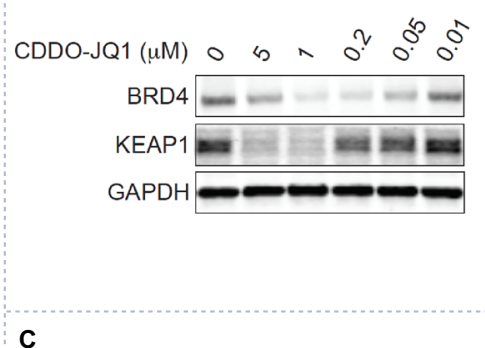

C
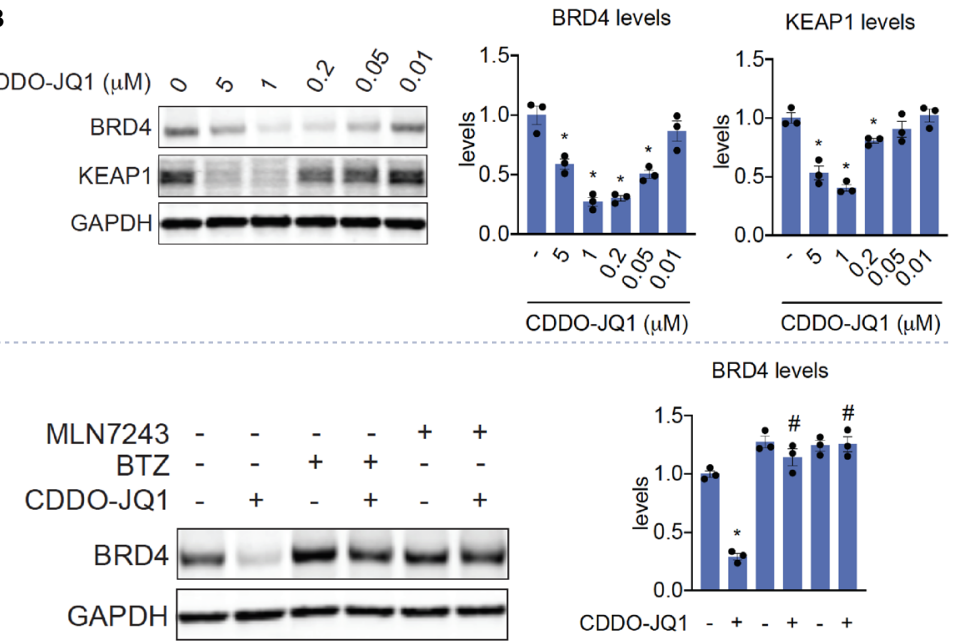

CDDO-JQ1 - + + + +

$\mathrm{BTZ}-\mathrm{C}_{-}+-$

MLN7243 - - - + +

BRD4 levels

D
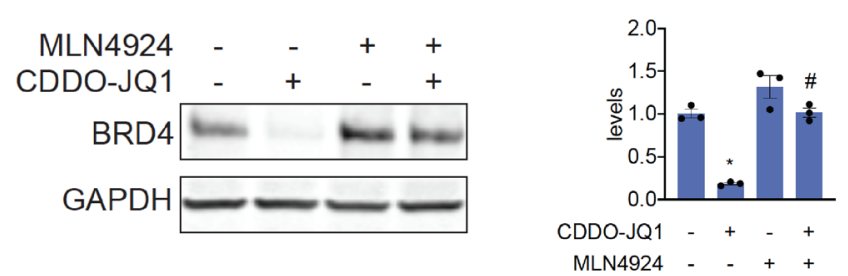

Figure 2. Bardoxolone-based protein degradation. (A) Synthesis of CDDO-JQ1. (B) Effect of CDDO-JQ1 on BRD4 and KEAP1 levels in 231MFP cells treated with DMSO vehicle or CDDO-JQ1 for $12 \mathrm{~h}$, assessed by Western blotting. (C) BRD4 levels in 231MFP cells pre-treated with vehicle, proteasome inhibitor bortezomib (BTZ) $(1 \mu \mathrm{M})$, or E1 activating enzyme inhibitor MLN7243 $(1 \mu \mathrm{M})$ for 30 min prior to treatment with vehicle or CDDO-JQ1 (200 nM) for $12 \mathrm{~h}$. (D) BRD4 levels in 231MFP cells pre-treated with DMSO vehicle or NEDD8 inhibitor MLN4924 $(1 \mu \mathrm{M})$ for 30 min prior to treatment with DMSO vehicle or CDDO-JQ1 (200 nM) for $12 \mathrm{~h}$. Blots are representative of $\mathrm{n}=3$ biological replicates/group. Data in bar graphs is expressed as individual replicate values and average \pm sem. Significance shown as ${ }^{*} \mathrm{p}<0.05$ compared to vehicle-treated control groups and ${ }^{\#} \mathrm{p}<0.05$ compared to CDDO-JQ1-treated groups.

\section{Results and discussion}

We were intrigued by the possibility of exploiting the triterpene derivative bardoxolone methyl (CDDO-Me) as a covalent, reversible ligase recruiter owing to its reversible interactions with cysteines on the E3 ligase KEAP1 and its highly electron-deficient $\alpha$-cyanoenone moiety (Fig. 2$)^{28-30}$. To test whether bardoxolone could be used as a novel E3 ligase recruiter, we synthesized a bardoxolone-based PROTAC by linking bardoxolone to the BET family bromodomain protein inhibitor JQ1 (Fig. 2A). The degradation of BET proteins has arisen as a powerful anticancer therapeutic strategy ${ }^{1,14,31,31}$, and the small molecule JQ1 has emerged as a general and robust ligand for use in PROTAC development toward these endeavors ${ }^{32}$. The free acid of JQ1 (1) was coupled with Boc-protected 


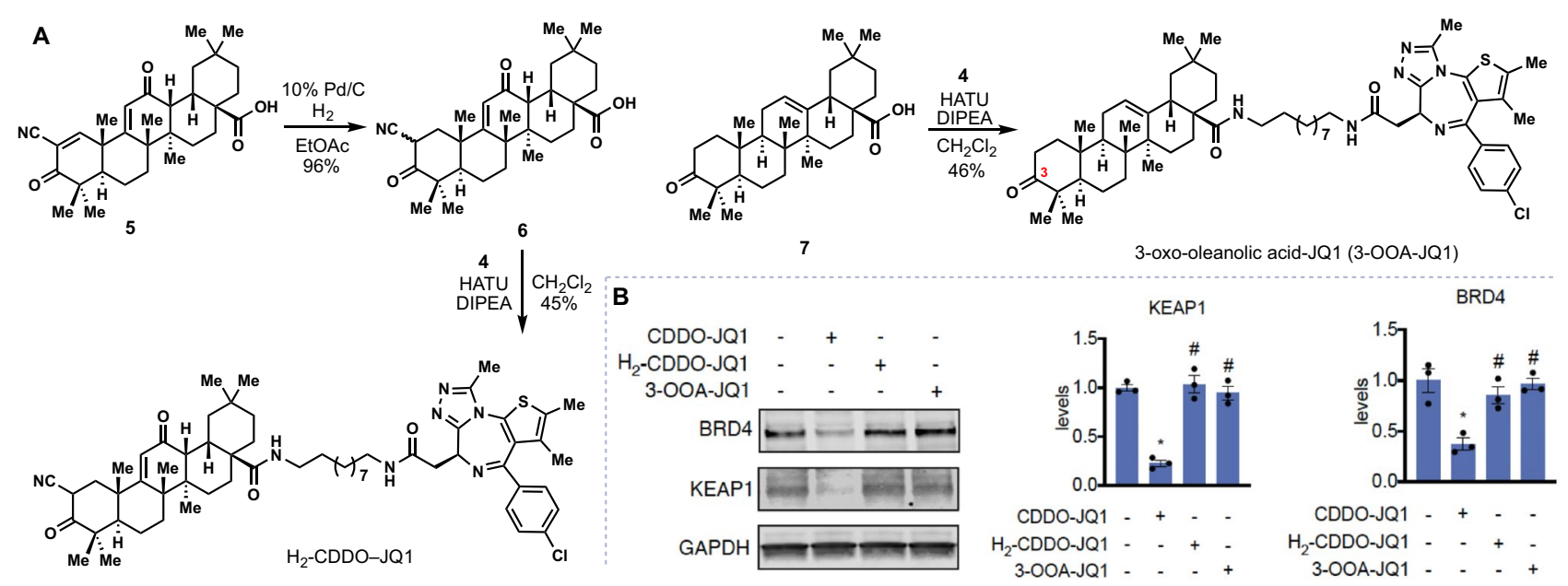

Figure 3. Degraders derived from unreactive variants of CDDO do not support the degradation of BRD4. (A) Synthesis of $\mathrm{H}_{2}$-CDDO-JQ1 and 3-oxo-oleanoic acid-JQ1 (3-OOA-JQ1). (B) Effect of CDDO-JQ1, $\mathrm{H}_{2}$-CDDO-JQ1, and 3-OOA-JQ1degraders on BRD4 and KEAP1 levels in 231MFP cells assessed by Western blotting. DMSO vehicle or compounds were treated at $1 \mu \mathrm{M}$ for $12 \mathrm{~h}$. Blots are representative of $\mathrm{n}=3$ biological replicates/group. Data in bar graphs is expressed as individual replicate values and average \pm sem. Significance shown as ${ }^{*} \mathrm{p}<0.05$ compared to vehicle-treated control groups and ${ }^{\#} \mathrm{p}<0.05$ compared to CDDO-JQ1-treated groups.

amine $\mathbf{2}$ and the resulting amide (see 3) converted into TFA salt $\mathbf{4}$ which was used without further purification ${ }^{33,34}$. Amide bond formation between $\mathbf{4}$ and CDDO then delivered the bifunctional degrader CDDO-JQ1.

We tested CDDO-JQ1 in the 231MFP human breast cancer cell line and observed dose-responsive degradation of BRD4; at higher concentrations loss of BRD4 degradation was observed, presumably due to the "hook" effect, a phenomenon not unexpected with a highly-reversible, covalently-binding bifunctional molecule (Fig. 2B). Interestingly, we also observed loss of KEAP1 at higher concentrations in cells treated with CDDO-JQ1, a finding that has been reported previously by treating cells with electrophilic stressors (Fig. 2B) ${ }^{35}$. Notably extensive degradation of BRD4 was observed in the 100-200 nM range without any optimization of linker length or composition ${ }^{36}$.

We further demonstrated that the CDDO-JQ1 mediated degradation of BRD4 was attenuated by pre-treatment with the proteasome inhibitor bortezomib as well as the E1 activating enzyme inhibitor MLN7243 (Fig. 2C). Given that KEAP1 belongs to the CUL3 family of E3 ligases that require NEDDylation for activity, we further demonstrated that the BRD4 degradation conferred by CDDO-JQ1 was also significantly attenuated by the NEDD8 activating enzyme inhibitor MLN4924 (Fig. 2D).

To show that the observed degradation by CDDO-JQ1 was not due to hydrophobic tagging of BRD4 leading to local protein unfolding and subsequent ubiquitination and degradation ${ }^{38-41}$, we synthesized two negative control compounds, both having significantly altered electrophilicity and resultant covalent protein reactivity, but crucially having similar physicochemical properties (Fig. 3A). First, hydrogenation of $\mathbf{5}\left(\mathrm{H}_{2}, \mathrm{Pd} / \mathrm{C}\right)$ generated ketone $\mathbf{6}$ as a mixture of diastereomers and enol tautomers. Coupling of this material with $\mathbf{4}$ then generated $\mathrm{H}_{2}$-CDDO-JQ1. Importantly, $\mathrm{H}_{2}$-CDDO-JQ1 did not induce BRD4 degradation in comparison to CDDO-JQ1 (Fig. 3B). Similarly, we prepared 3-oxo-oleanolic acid-JQ1 (3-OOA-JQ1), which possesses no potentially reactive alkenes of any type by coupling $7^{42}$ with 4 and found it also did not degrade BRD4 as compared to CDDO-JQ1.

Finally, we prepared the des-cyano variant of CDDO-JQ1 by coupling $8^{43}$ and 4 (Fig. 4A), and found that this compound (de-CN-CDDO-JQ1) also does not degrade BRD4 in 231 MFP cells indicating the criticality of the entire a-cyanoenone motif to this process (Fig. 4B). Interestingly, this chemical modification had also previously reduced the activity of bardoxolone methyl $\sim 1,000$ fold as an anti-inflammatory agent ${ }^{30}$. In general, all three of the changes made to the CDDO portion of CDDO-JQ1 (Figs. 3 and 4) were known to greatly reduce Nrf2 activation in the medicinal chemistry campaigns involving bardoxolone methyl as an anti-inflammatory drug ${ }^{28-30}$.

\section{Conclusion}

In summary, the first bifunctional protein degrader (PROTAC) based on the known KEAP1 ligand bardoxolone (CDDO) is reported. While robust, proteasome-dependent degradation of BRD4 was observed, a number of mechanistic questions remain: firstly, CDDO is not known to bind the Kelch domain of the KEAP1/Cul3 complex, the key area for recognition of Nrf2 and where degrader ${ }^{44}$, and multiple small molecule inhibitors presumably bind ${ }^{45-48}$. While a crystal structure of CDDO bound to Cys-151 of the BTB domain of KEAP1 has been solved, various reports have also posited that multiple cysteines are targeted by this class of compounds during the Nrf2 activation process in cells ${ }^{49-53}$. This raises the question how (or if) a CDDO-based PROTAC can mechanistically induce neosubstrate degradation in a KEAP1-dependent manner. Secondly, while bardoxolone is thought to activate Nrf2 through the targeting of reactive cysteines on KEAP1, it also interacts with additional pharmacological targets, including IKKb which modulates NF-kB signaling ${ }^{54}, \mathrm{mTOR}^{55}$, and others ${ }^{55,56}$. It should be noted that the direct detection of all proteome-wide targets of CDDO by pulldown studies has proven 
A
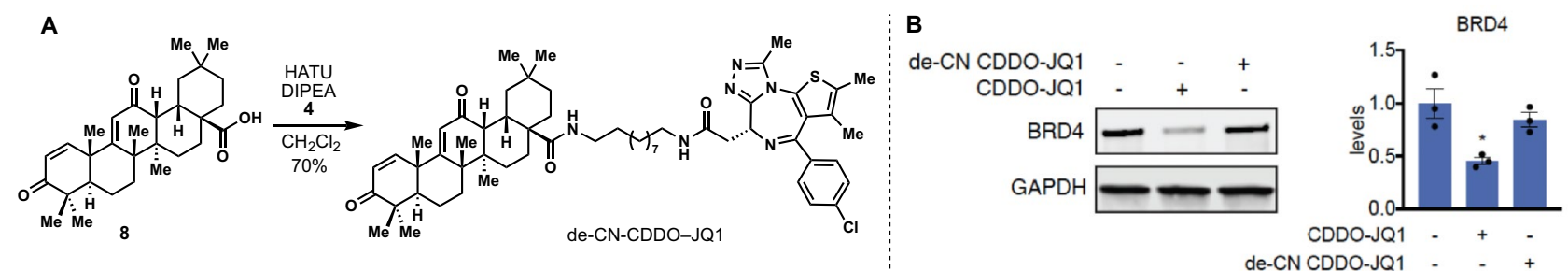

Figure 4. Functional significance of the a-cyanoenone moiety. (A) Synthesis of de-CN-CDDO-JQ1. (B) Effect of CDDO-JQ1 and de-CN-CDDO-JQ1 on BRD4 and GAPDH levels in 231MFP cells assessed by Western blotting. DMSO vehicle or compounds were treated at $1 \mu \mathrm{M}$ for $12 \mathrm{~h}$. Blots are representative of $\mathrm{n}=3$ biological replicates/group. Data in bar graphs is expressed as individual replicate values and average \pm sem. Significance shown as ${ }^{*} \mathrm{p}<0.05$ compared to vehicle-treated control groups and ${ }^{*} \mathrm{p}<0.05$ compared to CDDO-JQ1-treated groups.

challenging given the highly reversible nature of its cysteine interactions $s^{30,50,53,54-56}$. Thus, we cannot rule out at this moment that the degradation observed here may be due to one (or more) other cullin-family E3 ligases that are targeted by CDDO-JQ1.

Emerging evidence suggests that clinical resistance to PROTACs can occur through rewiring of the cellular E3 ligase machinery ${ }^{57,58}$, thus highlighting the critical need for more and diverse E3 ligase recruiters. Our combined results reported herein strongly implicate E3 ligase involvement and covalent cysteine reactivity in the mechanism of BRD4 degradation by CDDO-JQ1. Future chemoproteomic and genetic studies to map the proteome-wide targets of CDDO-JQ1 will be revealing in further understanding the mechanism of degradation reported herein. Nevertheless, the degradation toolkit has now been expanded to include CDDO as an easily prepared recruiter, and reversible, covalent E3 engagement as a promising concept for future TPD applications.

\section{Experimental section}

General synthetic methods. Unless otherwise noted, all reactions were performed in flame-dried glassware under a positive pressure of nitrogen or argon. Air- and moisture-sensitive liquids were transferred via syringe. Dry dichloromethane $\left(\mathrm{CH}_{2} \mathrm{Cl}_{2}\right)$ and $\mathrm{N}, \mathrm{N}$-dimethylformamide (DMF) were obtained by passing these previously degassed solvents through activated alumina columns. Bardoxolone methyl (CDDO-Me) was purchased from Sigma-Aldrich. Oleanolic acid was purchased from Acros Organics. (+)-JQ1 was purchased from Enovation Chemicals. All reagents were used as received from commercial sources, unless stated otherwise. Reactions were monitored by thin layer chromatography (TLC) on TLC silica gel $60 \mathrm{~F}_{254}$ glass plates (EMD Millipore) and visualized by UV irradiation and staining with $p$-anisaldehyde, phosphomolybdic acid, or Ninhydrin. Volatile solvents were removed under reduced pressure using a rotary evaporator. Flash column chromatog-

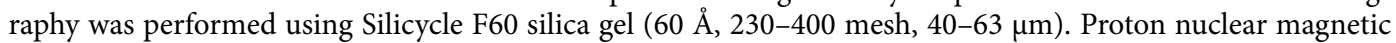
resonance $\left({ }^{1} \mathrm{H} \mathrm{NMR}\right)$ and carbon nuclear magnetic resonance $\left({ }^{13} \mathrm{C} \mathrm{NMR}\right)$ spectra were recorded on Bruker AV-600 and AV-700 spectrometers operating at 600 and $700 \mathrm{MHz}$ for ${ }^{1} \mathrm{H} \mathrm{NMR}$, and 151 and $176 \mathrm{MHz}$ for ${ }^{13} \mathrm{C}$ NMR (Supplementary Information). Chemical shifts are reported in parts per million (ppm) with respect to the residual solvent signal $\mathrm{CDCl}_{3}\left({ }^{1} \mathrm{H}\right.$ NMR: $\delta 7.26 ;{ }^{13} \mathrm{C}$ NMR: $\left.\delta 77.16\right), \mathrm{CD}_{2} \mathrm{Cl}_{2}\left({ }^{1} \mathrm{H}\right.$ NMR: $\delta 5.32 ;{ }^{13} \mathrm{C}$ NMR: $\delta$ 53.84). Peak multiplicities are reported as follows: $s=$ singlet, $d=$ doublet, $t=$ triplet, $d d=$ doublet of doublets, $t t=$ triplet of triplets, $m=$ multiplet, $b r=$ broad signal. IR spectra were recorded on a Bruker Vertex80 FTIR spectrometer. High-resolution mass spectra (HRMS) were obtained by the QB3/chemistry mass spectrometry facility at the University of California, Berkeley using a Thermo LTQ-FT mass spectrometer with electrospray ionization (ESI) technique.

Synthesis of compound 3: To a $10 \mathrm{~mL}$ reaction tube was added $\mathbf{1}^{33}(21.7 \mathrm{mg}, 0.0541 \mathrm{mmol}, 1.0$ equiv) and DMF $(1.0 \mathrm{~mL})$. DIPEA (37.7 $\mu \mathrm{L}, 0.216 \mathrm{mmol}, 4.0$ equiv) and HATU (22.6 mg, $0.0595 \mathrm{mmol}, 1.1$ equiv) were added at $0{ }^{\circ} \mathrm{C}$ and the reaction mixture was stirred at room temperature for $30 \mathrm{~min}$. The reaction mixture was then cooled back to $0^{\circ} \mathrm{C}$ and amine $2^{34}(29.5 \mathrm{mg}, 0.108 \mathrm{mmol}, 2.0$ equiv) was added. The resulting mixture was allowed to stir at room temperature for $1 \mathrm{~h}$ before quenching with saturated aq. $\mathrm{NH}_{4} \mathrm{Cl}(30 \mathrm{~mL})$. The layers were separated, and the aqueous phase was extracted with EtOAc $(3 \times 5 \mathrm{~mL})$. The combined organic layer was dried over $\mathrm{Na}_{2} \mathrm{SO}_{4}$ and concentrated in vacuo. The residue was purified by column chromatography (3\% $\left.\mathrm{MeOH} / \mathrm{CH}_{2} \mathrm{Cl}_{2}\right)$ to afford compound $3(30.0 \mathrm{mg}, 84 \%$ yield $)$ as a yellow oil: ${ }^{1} \mathrm{H} \mathrm{NMR}\left(600 \mathrm{MHz}, \mathrm{CDCl}_{3}\right) \delta 7.40$ $(\mathrm{d}, J=8.1 \mathrm{~Hz}, 2 \mathrm{H}), 7.32(\mathrm{~d}, J=8.2 \mathrm{~Hz}, 2 \mathrm{H}), 6.39(\mathrm{br}, 1 \mathrm{H}), 4.61(\mathrm{t}, J=7.0 \mathrm{~Hz}, 1 \mathrm{H}), 4.51(\mathrm{br}, 1 \mathrm{H}), 3.55(\mathrm{dd}, J=14.0$, $7.5 \mathrm{~Hz}, 1 \mathrm{H}), 3.39-3.27(\mathrm{~m}, 2 \mathrm{H}), 3.22(\mathrm{dt}, J=13.2,6.6 \mathrm{~Hz}, 1 \mathrm{H}), 3.10(\mathrm{br}, 2 \mathrm{H}), 2.66(\mathrm{~s}, 3 \mathrm{H}), 2.40(\mathrm{~s}, 3 \mathrm{H}), 1.67(\mathrm{~s}, 3 \mathrm{H})$, $1.56-1.42(\mathrm{~m}, 4 \mathrm{H}), 1.44(\mathrm{~s}, 9 \mathrm{H}), 1.33-1.22(\mathrm{~m}, 12 \mathrm{H}) ;{ }^{13} \mathrm{C}$ NMR $\left(151 \mathrm{MHz}, \mathrm{CDCl}_{3}\right) \delta 170.5,164.0,156.1,155.8$, $150.0,136.9,136.8,132.3,131.1,130.9,130.6,129.9,128.9,79.1,54.7,40.8,39.85,39.77,30.2,29.68,29.61,29.59$, $29.41,29.40,28.6,27.1,26.9,14.5,13.2,12.0$; IR (thin film) vmax 3,347, 2,956, 2,924, 2,853, 1721, 1,453, 1,376, $1,274,1,176,1,110,714 \mathrm{~cm}^{-1}$; HRMS (ESI) calcd. for $\left[\mathrm{C}_{34} \mathrm{H}_{48} \mathrm{O}_{3} \mathrm{~N}_{6} \mathrm{ClS}\right]^{+}\left([\mathrm{M}+\mathrm{H}]^{+}\right): m / z 655.3192$, found: 655.3180 .

Synthesis of CDDO-JQ1: $i$. To a $10 \mathrm{~mL}$ reaction tube was added compound $3(24.3 \mathrm{mg}, 0.037 \mathrm{mmol}, 1.0$ equiv), triethylsilane $\left(12.0 \mu \mathrm{L}, 0.074 \mathrm{mmol}, 2.0\right.$ equiv) and $\mathrm{CH}_{2} \mathrm{Cl}_{2}(0.6 \mathrm{~mL})$. Trifluoroacetic acid $(0.2 \mathrm{~mL})$ was added slowly at $0{ }^{\circ} \mathrm{C}$. The reaction mixture was allowed to warm up to room temperature and stirred for $3 \mathrm{~h}$. After completion of the reaction, the mixture was concentrated by rotatory evaporation. The residue was dried under high vacuum for $3 \mathrm{~h}$ to provide crude compound $\mathbf{4}$ as a yellow oil, which was used directly in the next step without further purification. 
ii. To a $10 \mathrm{~mL}$ reaction tube was added CDDO (5) $(12.7 \mathrm{mg}, 0.026 \mathrm{mmol}, 1.0$ equiv), HATU (10.3 mg, $0.027 \mathrm{mmol}, 1.05$ equiv), DIPEA $\left(13.5 \mu \mathrm{L}, 0.078 \mathrm{mmol}, 3.0\right.$ equiv) and $\mathrm{CH}_{2} \mathrm{Cl}_{2}(0.4 \mathrm{~mL})$. After stirring at room temperature for $12 \mathrm{~h}$, the reaction was quenched with saturated aq. $\mathrm{NH}_{4} \mathrm{Cl}$ and extracted with $\mathrm{CH}_{2} \mathrm{Cl}_{2}(3 \times 1 \mathrm{~mL})$. The combined organic layer was dried over $\mathrm{Na}_{2} \mathrm{SO}_{4}$ and concentrated in vacuo. The resulting activated ester was then transferred into another reaction tube containing the previously prepared crude compound $4(0.037 \mathrm{mmol}$, 1.4 equiv). $\mathrm{CH}_{2} \mathrm{Cl}_{2}(0.4 \mathrm{~mL})$ and DIPEA $(22.5 \mu \mathrm{L}, 0.129 \mathrm{mmol}, 5.0$ equiv) was added and the reaction mixture was stirred at room temperature for $12 \mathrm{~h}$ before quenching with saturated aq. $\mathrm{NH}_{4} \mathrm{Cl}$. The layers were separated, and the aqueous phase was extracted with $\mathrm{CH}_{2} \mathrm{Cl}_{2}(3 \times 1 \mathrm{~mL})$. The combined organic layer was dried over $\mathrm{Na}_{2} \mathrm{SO}_{4}$ and concentrated in vacuo. The residue was purified by column chromatography $\left(4 \% \mathrm{MeOH} / \mathrm{CH}_{2} \mathrm{Cl}_{2}\right)$ followed by preparative TLC (5\% $\left.\mathrm{MeOH} / \mathrm{CHCl}_{3}\right)$ to afford degrader CDDO-JQ1 (12.8 $\mathrm{mg}, 48 \%$ yield) as a colorless oil which slowly solidifies: ${ }^{1} \mathrm{H}$ NMR $\left(700 \mathrm{MHz}, \mathrm{CD}_{2} \mathrm{Cl}_{2}\right) \delta 8.09(\mathrm{~s}, 1 \mathrm{H}), 7.43(\mathrm{~d}, J=8.3 \mathrm{~Hz}, 2 \mathrm{H}), 7.34(\mathrm{~d}, J=8.3 \mathrm{~Hz}$, $2 \mathrm{H}), 6.49(\mathrm{t}, J=5.9 \mathrm{~Hz}, 1 \mathrm{H}), 5.95(\mathrm{~s}, 1 \mathrm{H}), 5.85(\mathrm{t}, J=5.8 \mathrm{~Hz}, 1 \mathrm{H}), 4.57(\mathrm{t}, J=6.9 \mathrm{~Hz}, 1 \mathrm{H}), 3.42(\mathrm{dd}, J=14.3,7.2 \mathrm{~Hz}$, $1 \mathrm{H}), 3.32-3.15(\mathrm{~m}, 5 \mathrm{H}), 3.08(\mathrm{~d}, J=4.6 \mathrm{~Hz}, 1 \mathrm{H}), 2.89-2.84(\mathrm{~m}, 1 \mathrm{H}), 2.63(\mathrm{~s}, 3 \mathrm{H}), 2.40(\mathrm{~s}, 3 \mathrm{H}), 2.00(\mathrm{td}, J=14.0$, $3.9 \mathrm{~Hz}, 1 \mathrm{H}), 1.81-1.69(\mathrm{~m}, 5 \mathrm{H}), 1.67(\mathrm{~s}, 3 \mathrm{H}), 1.57-1.47(\mathrm{~m}, 6 \mathrm{H}), 1.46(\mathrm{~s}, 3 \mathrm{H}), 1.45-1.39(\mathrm{~m}, 1 \mathrm{H}), 1.36-1.19(\mathrm{~m}$, $16 \mathrm{H}), 1.32(\mathrm{~s}, 3 \mathrm{H}), 1.23(\mathrm{~s}, 3 \mathrm{H}), 1.21-1.15(\mathrm{~m}, 1 \mathrm{H}), 1.14(\mathrm{~s}, 3 \mathrm{H}), 1.00(\mathrm{~s}, 3 \mathrm{H}), 0.96(\mathrm{~s}, 3 \mathrm{H}), 0.89(\mathrm{~s}, 3 \mathrm{H}) ;{ }^{13} \mathrm{C} \mathrm{NMR}$ $\left(151 \mathrm{MHz}, \mathrm{CD}_{2} \mathrm{Cl}_{2}\right) \delta 199.4,197.4,177.0,170.4,168.9,166.6,164.2,156.2,150.4,137.3,136.9,132.8,131.4,131.2$, $130.7,130.4,129.0,124.4,115.1,114.7,54.9,49.8,48.0,46.7,46.3,45.4,43.0,42.5,39.97,39.89,39.78,36.4,35.0$, $34.5,33.4,32.19,32.07,30.9,30.2,30.1,30.0,29.83,29.81,29.65,29.65,28.2,27.4,27.3,27.0,26.7,25.0,23.6$, 23.3, 21.8, 18.6, 14.6, 13.2, 12.0; IR (thin film) vmax 3,346, 2,924, 2,852, 1,715, 1,661, 1,593, 1,562, 1,532, 1,465, $1,383,1,262,1,091,1,015,805 \mathrm{~cm}^{-1}$; HRMS (ESI) calcd for $\left[\mathrm{C}_{60} \mathrm{H}_{79} \mathrm{O}_{4} \mathrm{~N}_{7} \mathrm{ClS}\right]^{+}\left([\mathrm{M}+\mathrm{H}]^{+}\right): m / z 1,028.5597$, found: $1,028.5599$.

Synthesis of Compound 6: To a solution of CDDO (5) $(5.8 \mathrm{mg}, 0.012 \mathrm{mmol}, 1.0$ equiv) in EtOAc ( $1 \mathrm{~mL})$ was added $10 \% \mathrm{Pd} / \mathrm{C}(5 \mathrm{mg})$ in one portion. Hydrogen gas was bubbled through the reaction mixture via a long steel needle that was attached to a hydrogen balloon. After $5 \mathrm{~min}$, the needle was lifted above the solvent level and the reaction was stirred for additional 20 min under $\mathrm{H}_{2}$ atmosphere. The reaction mixture was filtered through celite, washed with EtOAc $(2 \mathrm{~mL})$ and concentrated in vacuo to afford compound 6 (5.6 mg, 96\% yield) as a white solid, which was pure enough $\left(>95 \%\right.$ by ${ }^{1} \mathrm{H}$ NMR) to be used in the next step without further purification. The product exists as a mixture of diastereomers and enol/keto tautomers (all peaks are listed): ${ }^{1} \mathrm{H}$ NMR $\left(600 \mathrm{MHz}, \mathrm{CDCl}_{3}\right) \delta 5.77(\mathrm{~s}, 0.65 \mathrm{H}), 5.74(\mathrm{~s}, 0.21 \mathrm{H}), 5.72(\mathrm{~s}, 0.14 \mathrm{H}), 3.98(\mathrm{dd}, J=13.8,5.4 \mathrm{~Hz}, 0.21 \mathrm{H}), 3.93$ $(\mathrm{dd}, J=11.7,8.1 \mathrm{~Hz}, 0.14 \mathrm{H}), 3.06-2.98(\mathrm{~m}, 1 \mathrm{H}), 2.98-2.90(\mathrm{~m}, 1 \mathrm{H}), 2.71-2.59(\mathrm{~m}, 0.35 \mathrm{H}), 2.42(\mathrm{~d}, J=15.0 \mathrm{~Hz}$, $0.65 \mathrm{H}), 2.37(\mathrm{dd}, J=13.5,8.1 \mathrm{~Hz}, 0.14 \mathrm{H}), 2.25(\mathrm{~d}, J=15.0 \mathrm{~Hz}, 0.65 \mathrm{H}), 2.03(\mathrm{t}, J=13.5 \mathrm{~Hz}, 0.21 \mathrm{H}), 1.98-1.16(\mathrm{~m}$, $24 \mathrm{H}), 1.15(\mathrm{~s}, 0.42 \mathrm{H}), 1.14(\mathrm{~s}, 0.63 \mathrm{H}), 1.12(\mathrm{~s}, 1.95 \mathrm{H}), 1.05(\mathrm{~s}, 0.42 \mathrm{H}), 1.00(\mathrm{~s}, 4.95 \mathrm{H}), 0.99(\mathrm{~s}, 0.63 \mathrm{H}), 0.90(\mathrm{~s}$, $3 \mathrm{H}) ;{ }^{13} \mathrm{C} \mathrm{NMR}\left(151 \mathrm{MHz}, \mathrm{CDCl}_{3}\right) \delta 206.3,204.1,199.7,199.5,199.1,183.2,182.9,175.3,175.1,173.7,171.5$, $126.4,124.6,123.7,118.7,117.1,116.6,79.2,51.8,49.8,49.72,49.70,49.4,48.6,48.5,47.2,47.13,47.11,47.06$, $45.7,45.6,45.4,42.9,42.3,42.04,42.00,41.9,39.6,38.9,38.73,38.69,38.2,37.5,36.0,35.9,35.8,35.7,34.6,34.5$, $33.4,33.1,33.0,32.5,31.59,31.58,31.3,30.80,30.77,30.5,29.8,28.9,28.3,28.2,28.1,28.0,26.9,25.1,24.3,24.1$, 23.22, 23.18, 23.16, 23.12, 22.8, 22.7, 22.6, 21.8, 21.71, 21.68, 21.57, 20.01, 19.96, 19.3, 18.94, 18.88; IR (thin film) 2,927, 2,856, 1,722, 1,694, 1,662, 1,466, 1,385, 1,261, 1,241, 1,186, 1,161, 883, 807, $750 \mathrm{~cm}^{-1}$; HRMS (ESI) calcd for $\left[\mathrm{C}_{31} \mathrm{H}_{42} \mathrm{O}_{4} \mathrm{~N}\right]^{-}\left([\mathrm{M}-\mathrm{H}]^{-}\right): m / z 492.3119$, found: 492.3120 .

Synthesis of $\mathbf{H}_{2}$-CDDO-JQ1: To a $10 \mathrm{~mL}$ reaction tube was added compound $\mathbf{6}$ (5.6 mg, $0.011 \mathrm{mmol}, 1.0$ equiv), HATU ( $4.7 \mathrm{mg}, 0.012 \mathrm{mmol}, 1.1$ equiv), DIPEA $\left(5.9 \mu \mathrm{L}, 0.034 \mathrm{mmol}, 3.0\right.$ equiv) and $\mathrm{CH}_{2} \mathrm{Cl}_{2}(0.4 \mathrm{~mL})$. After stirring at room temperature for $12 \mathrm{~h}$, the reaction was quenched with saturated aq. $\mathrm{NH}_{4} \mathrm{Cl}$ and extracted with $\mathrm{CH}_{2} \mathrm{Cl}_{2}(3 \times 1 \mathrm{~mL})$. The combined organic layer was dried over $\mathrm{Na}_{2} \mathrm{SO}_{4}$ and concentrated in vacuo. The resulting activated ester was then transferred into another reaction tube containing the previously prepared crude compound 4 ( $0.017 \mathrm{mmol}, 1.5$ equiv). $\mathrm{CH}_{2} \mathrm{Cl}_{2}(0.4 \mathrm{~mL})$ and DIPEA (9.8 $\mu \mathrm{L}, 0.056 \mathrm{mmol}, 5.0$ equiv) was added and the reaction mixture was stirred at room temperature for $12 \mathrm{~h}$ before quenching with saturated $a q$. $\mathrm{NH}_{4} \mathrm{Cl}$. The layers were separated, and the aqueous phase was extracted with $\mathrm{CH}_{2} \mathrm{Cl}_{2}(3 \times 1 \mathrm{~mL})$. The combined organic layer was dried over $\mathrm{Na}_{2} \mathrm{SO}_{4}$ and concentrated in vacuo. The residue was purified by preparative TLC (5\% $\mathrm{MeOH} / \mathrm{CHCl}_{3}$ ) to afford $\mathbf{H}_{2}$-CDDO-JQ1 $(5.2 \mathrm{mg}, 45 \%$ yield) as a colorless oil which slowly solidifies (mixture of diasteromers and enol/keto tautomers): ${ }^{1} \mathrm{H} \mathrm{NMR}\left(600 \mathrm{MHz}, \mathrm{CD}_{2} \mathrm{Cl}_{2}\right) \delta 7.43(\mathrm{~d}, J=8.4 \mathrm{~Hz}, 2 \mathrm{H}), 7.35(\mathrm{~d}, J=8.3 \mathrm{~Hz}$, $2 \mathrm{H}), 6.47(\mathrm{t}, J=5.8 \mathrm{~Hz}, 0.6 \mathrm{H}), 6.47(\mathrm{t}, J=5.8 \mathrm{~Hz}, 0.4 \mathrm{H}), 5.91-5.80(\mathrm{~m}, 1 \mathrm{H})$, ), $5.74(\mathrm{~s}, 0.4 \mathrm{H}), 5.72(\mathrm{~s}, 0.4 \mathrm{H}), 5.69$ $(\mathrm{s}, 0.2 \mathrm{H}), 4.62-4.54(\mathrm{~m}, 1 \mathrm{H}), 4.04(\mathrm{dd}, J=14.1,5.1 \mathrm{~Hz}, 0.4 \mathrm{H}), 3.96(\mathrm{dd}, J=12.0,7.8 \mathrm{~Hz}, 0.2 \mathrm{H}), 3.49-3.39(\mathrm{~m}, 1 \mathrm{H})$, 3.34-3.14 (m, 5H), 3.07-2.99 (m, 1H), 2.89-2.81 (m, 1H), 2.70-1.18 (m, 48H), 1.18-1.14 (m, 3H), 1.13-1.10 (m, $3 \mathrm{H}), 1.05-0.95(\mathrm{~m}, 6 \mathrm{H}), 0.92-0.87(\mathrm{~m}, 3 \mathrm{H}) ;{ }^{13} \mathrm{C} \mathrm{NMR}\left(151 \mathrm{MHz}, \mathrm{CD}_{2} \mathrm{Cl}_{2}\right) \delta 207.3,205.0,200.0,199.6,177.10$, $177.06,175.9,175.5,174.2,171.4,170.43,170.35,164.24,164.18,156.15,156.10,150.5,150.4,137.29,137.26$, $136.92,136.89,132.8,132.7,131.5,131.4,131.17,131.15,130.8,130.7,130.4,129.0,126.6,124.6,123.7,119.1$, $117.7,117.3,80.1,54.92,54.90,52.0,49.8,49.66,49.64,49.4,48.8,47.3,46.8,46.75,46.73,46.1,46.0,45.8,43.3$, $42.7,42.25,42.23,42.1,39.95,39.89,39.88,39.8,39.10,39.05,38.99,38.8,38.0,36.6,36.5,36.4,36.1,35.02,34.99$, $34.6,33.45,33.43,32.8,32.3,32.2,31.6,30.95,30.92$, 30.8, 30.30, 30.26, 30.15, 30.10, 30.05, 30.02, 29.99, 29.91, $29.83,29.80,29.73,29.67,29.64,28.9,28.3,28.24,28.16,27.9,27.6,27.43,27.40,27.3,27.0,24.9,24.6,24.3,23.65$, $23.54,23.51,23.48,23.33,23.29,23.2,21.9,21.80,21.75,21.68,20.0,19.9,19.6,19.22,19.15,14.6,13.2,12.0$; IR (thin film) vmax 3,345, 2,924, 2,853, 1,728, 1,660, 1,595, 1532, 1,465, 1,417, 1,382, 1,091, 1,015, 840, 805, 721 cm HRMS (ESI) calcd. for $\left[\mathrm{C}_{60} \mathrm{H}_{81} \mathrm{O}_{4} \mathrm{~N}_{7} \mathrm{ClS}\right]^{+}\left([\mathrm{M}+\mathrm{H}]^{+}\right): \mathrm{m} / z 1,030.5754$, found: $1,030.5744$.

3-Oxo-oleanolic acid-JQ1 (3-OOA-JQ1): to a $10 \mathrm{~mL}$ reaction tube was added compound $7^{42}(9.3 \mathrm{mg}$, $0.0204 \mathrm{mmol}, 1.0$ equiv) and $\mathrm{CH}_{2} \mathrm{Cl}_{2}(1.0 \mathrm{~mL})$. The resulting solution was cooled to $0{ }^{\circ} \mathrm{C}$, and DIPEA $(14.3 \mu \mathrm{L}$, $0.0818 \mathrm{mmol}, 4.0$ equiv) and HATU $(8.5 \mathrm{mg}, 0.0225 \mathrm{mmol}, 1.1$ equiv) were added. The reaction mixture was allowed to stir at room temperature for $30 \mathrm{~min}$ before quenching with saturated aq. $\mathrm{NH}_{4} \mathrm{Cl}$. The layers were separated, and the aqueous phase was extracted with $\mathrm{CH}_{2} \mathrm{Cl}_{2}(3 \times 1 \mathrm{~mL})$. The combined organic layer was dried over $\mathrm{Na}_{2} \mathrm{SO}_{4}$ and concentrated in vacuo. The resulting activated ester was then transferred into another reaction 
tube containing previously prepared crude compound 4 (17.0 mg, $0.0307 \mathrm{mmol}, 1.5$ equiv), DIPEA ( $35.5 \mu \mathrm{L}$, $0.204 \mathrm{mmol}, 10.0$ equiv) and $\mathrm{CH}_{2} \mathrm{Cl}_{2}(1.0 \mathrm{~mL})$. The reaction mixture was allowed to stir at room temperature for $12 \mathrm{~h}$ before quenching with saturated aq. $\mathrm{NH}_{4} \mathrm{Cl}$. The layers were separated, and the aqueous phase was extracted with $\mathrm{CH}_{2} \mathrm{Cl}_{2}(3 \times 1 \mathrm{~mL})$. The combined organic layer was dried over $\mathrm{Na}_{2} \mathrm{SO}_{4}$ and concentrated in vacuo. The residue was purified by preparative TLC $\left(6 \% \mathrm{MeOH} / \mathrm{CH}_{2} \mathrm{Cl}_{2}\right.$, developed twice) to afford 3-oxo-oleanolic acid-JQ1 (9.3 mg, 46\% yield) as a white solid: ${ }^{1} \mathrm{H}$ NMR (600 MHz, $\left.\mathrm{CDCl}_{3}\right) \delta 7.42-7.38(\mathrm{~m}, 2 \mathrm{H}), 7.35-7.31(\mathrm{~m}$, $2 \mathrm{H}), 6.36(\mathrm{t}, J=5.7 \mathrm{~Hz}, 1 \mathrm{H}), 5.88(\mathrm{t}, J=5.5 \mathrm{~Hz}, 1 \mathrm{H}), 5.39(\mathrm{t}, J=3.6 \mathrm{~Hz}, 1 \mathrm{H}), 4.60(\mathrm{dd}, J=7.6,6.3 \mathrm{~Hz}, 1 \mathrm{H}), 3.55$ $(\mathrm{dd}, J=14.1,7.7 \mathrm{~Hz}, 1 \mathrm{H}), 3.39-3.28(\mathrm{~m}, 3 \mathrm{H}), 3.21(\mathrm{dq}, J=13.2,7.0 \mathrm{~Hz}, 1 \mathrm{H}), 3.06-2.97(\mathrm{~m}, 1 \mathrm{H}), 2.67(\mathrm{~s}, 3 \mathrm{H})$, 2.59-2.50 (m, 2H), $2.40(\mathrm{~d}, J=0.8 \mathrm{~Hz}, 3 \mathrm{H}), 2.37$ (ddd, $J=15.9,6.8,3.6 \mathrm{~Hz}, 1 \mathrm{H}), 2.03-1.92(\mathrm{~m}, 3 \mathrm{H}), 1.89$ (ddd, $J=13.2,7.3,3.7 \mathrm{~Hz}, 1 \mathrm{H}), 1.77(\mathrm{t}, J=13.3 \mathrm{~Hz}, 1 \mathrm{H}), 1.74-1.64(\mathrm{~m}, 3 \mathrm{H}), 1.67(\mathrm{~s}, 3 \mathrm{H}), 1.61-1.38(\mathrm{~m}, 12 \mathrm{H}), 1.36-1.18$ $(\mathrm{m}, 16 \mathrm{H}), 1.17(\mathrm{~s}, 3 \mathrm{H}), 1.09(\mathrm{~s}, 3 \mathrm{H}), 1.06(\mathrm{~s}, 3 \mathrm{H}), 1.05(\mathrm{~s}, 3 \mathrm{H}), 0.91(\mathrm{~s}, 3 \mathrm{H}), 0.91(\mathrm{~s}, 3 \mathrm{H}), 0.83(\mathrm{~s}, 3 \mathrm{H}) ;{ }^{13} \mathrm{C} \mathrm{NMR}$ $\left(151 \mathrm{MHz}, \mathrm{CDCl}_{3}\right) \delta 217.7,178.1,170.5,164.0,155.8,150.0,145.4,137.0,136.8,132.3,131.1,131.0,130.6,130.0$, $128.9,122.5,55.4,54.7,47.6,47.0,46.9,46.4,42.6,42.4,39.86,39.82,39.6,39.5,39.3,36.9,34.32,34.30,33.2$, $32.7,32.1,30.9,29.70,29.70,29.64,29.52,29.46,29.43,27.5,27.3,27.1,26.6,25.8,23.9,23.8,23.7,21.6,19.7$, 17.0, 15.2, 14.5, 13.2, 12.0. IR (thin film) vmax 3,346, 2,925, 2,854, 1,705, 1,654, 1593, 1532, 1,457, 1,419, 1,381, $1,274,1,176,1,110,1,091,1,014,839,805,749,714 \mathrm{~cm}^{-1}$; HRMS (ESI) calcd for $\left[\mathrm{C}_{59} \mathrm{H}_{84} \mathrm{O}_{3} \mathrm{~N}_{6} \mathrm{ClS}\right]^{+}\left([\mathrm{M}+\mathrm{H}]^{+}\right)$: $m / z$ 991.6009, found: 991.6005 .

De-CN-CDDO-JQ1: To a $10 \mathrm{~mL}$ reaction tube was added compound $\mathbf{8}^{43}$ ( $2.4 \mathrm{mg}, 0.0051 \mathrm{mmol}, 1.0$ equiv), HATU ( $2.2 \mathrm{mg}, 0.0057 \mathrm{mmol}, 1.1$ equiv), DIPEA $\left(2.6 \mu \mathrm{L}, 0.015 \mathrm{mmol}, 3.0\right.$ equiv) and $\mathrm{CH}_{2} \mathrm{Cl}_{2}(0.3 \mathrm{~mL})$. After stirring at room temperature for $12 \mathrm{~h}$, the reaction was quenched with saturated $a q . \mathrm{NH}_{4} \mathrm{Cl}$ and extracted with $\mathrm{CH}_{2} \mathrm{Cl}_{2}(3 \times 1 \mathrm{~mL})$. The combined organic layer was dried over $\mathrm{Na}_{2} \mathrm{SO}_{4}$ and concentrated in vacuo. The resulting activated ester was then transferred into another reaction tube containing the previously prepared crude compound 4 ( $0.0076 \mathrm{mmol}, 1.5$ equiv). $\mathrm{CH}_{2} \mathrm{Cl}_{2}(0.4 \mathrm{~mL})$ and DIPEA $(2.6 \mu \mathrm{L}, 0.015 \mathrm{mmol}, 3.0$ equiv) was added and the reaction mixture was stirred at room temperature for $12 \mathrm{~h}$ before quenching with saturated $a q$. $\mathrm{NH}_{4} \mathrm{Cl}$. The layers were separated, and the aqueous phase was extracted with $\mathrm{CH}_{2} \mathrm{Cl}_{2}(3 \times 1 \mathrm{~mL})$. The combined organic layer was dried over $\mathrm{Na}_{2} \mathrm{SO}_{4}$ and concentrated in vacuo. The residue was purified by preparative TLC (5\% $\mathrm{MeOH} / \mathrm{CHCl}_{3}$ ) to afford De-CN-CDDO-JQ1 (3.6 mg, 70\% yield) as a colorless oil which slowly solidifies: ${ }^{1} \mathrm{H}$ NMR $\left(600 \mathrm{MHz}, \mathrm{CD}_{2} \mathrm{Cl}_{2}\right) \delta 7.43(\mathrm{~d}, J=8.3 \mathrm{~Hz}, 2 \mathrm{H}), 7.38-7.31(\mathrm{~m}, 3 \mathrm{H}), 6.49(\mathrm{t}, J=5.9 \mathrm{~Hz}, 1 \mathrm{H}), 5.97(\mathrm{~s}, 1 \mathrm{H})$, $5.93-5.82(\mathrm{~m}, 2 \mathrm{H}), 4.56(\mathrm{t}, J=6.9 \mathrm{~Hz}, 1 \mathrm{H}), 3.42(\mathrm{dd}, J=14.3,7.2 \mathrm{~Hz}, 1 \mathrm{H}), 3.32-3.14(\mathrm{~m}, 5 \mathrm{H}), 3.03(\mathrm{~d}, J=4.6 \mathrm{~Hz}$, $1 \mathrm{H}), 2.91-2.84(\mathrm{~m}, 1 \mathrm{H}), 2.63(\mathrm{~s}, 3 \mathrm{H}), 2.40(\mathrm{~s}, 3 \mathrm{H}), 2.00(\mathrm{td}, J=13.9,3.9 \mathrm{~Hz}, 1 \mathrm{H}), 1.81-1.69(\mathrm{~m}, 5 \mathrm{H}), 1.68(\mathrm{~s}, 3 \mathrm{H})$, 1.56-1.42 (m, 7H), $1.40(\mathrm{~s}, 3 \mathrm{H}), 1.38-1.32(\mathrm{~m}, 2 \mathrm{H}), 1.31(\mathrm{~s}, 3 \mathrm{H}), 1.30-1.24(\mathrm{~m}, 14 \mathrm{H}), 1.23-1.17(\mathrm{~m}, 2 \mathrm{H}), 1.15$ (s, 3H), 1.08 (s, 3H), 1.00 (s, 3H), 0.96 (s, 3H), 0.89 (s, 3H); ${ }^{13} \mathrm{C}$ NMR $\left(151 \mathrm{MHz}, \mathrm{CD}_{2} \mathrm{Cl}_{2}\right) \delta 203.6,200.0,177.1$, $172.0,170.5,164.2,156.2,155.2,150.4,137.3,136.9,132.8,131.4,131.2,130.7,130.3,129.0,126.0,123.8,54.9$, $49.7,48.6,46.8,46.1,45.0,42.4,42.2,40.0,39.9,39.7,36.4,35.0,34.6,33.4,32.5,32.2,30.9,30.3,30.1,30.0,29.83$, $29.80,29.6,28.2,27.4,27.29,27.25,26.9,25.0,23.6,23.3,21.83,21.80,18.7,14.6,13.2,12.0$; IR (thin film) vmax 3,366, 2,925, 2,854, 1,659, 1,596, 1,535, 1,462, 1,382, 1,263, 1,093, 1,016, 846, 801, $605 \mathrm{~cm}^{-1}$; HRMS (ESI) calcd. for $\left[\mathrm{C}_{59} \mathrm{H}_{80} \mathrm{O}_{4} \mathrm{~N}_{6} \mathrm{ClS}\right]^{+}\left([\mathrm{M}+\mathrm{H}]^{+}\right): m / z 1,003.5645$, found: $1,003.5623$ (Supplementary Information).

General biological methods. Materials. Primary antibodies to BRD4 (Abcam, Ab128874), GAPDH (Proteintech Group Inc., 60004-1-Ig), and KEAP1 (Cell Signaling Technologies, D6B12) were obtained from commercial sources and dilutions were prepared according to manufacturer recommendations. Anti-rabbit and anti-mouse secondary antibodies were purchased from Licor (IRDye 800CW Goat anti-Rabbit IgG Secondary Antibody and IRDye 700CW Goat anti-Mouse IgG Secondary Antibody). The commercially available inhibitors bortezomib (Alfa Aesar J60378MA), MLN7243 (SelleckChem S8341) and MLN4924 (SelleckChem S7109) were purchased as solids and DMSO solutions were prepared at the appropriate concentrations.

Cell culture. 231MFP cells were obtained from Prof. B. Cravatt (Scripps) and were generated from explanted tumor xenografts of MDA-MB-231 cells as previously described ${ }^{59}$. They were cultured in L15 medium containing $10 \%(\mathrm{v} / \mathrm{v})$ fetal bovine serum (FBS), maintained at $37^{\circ} \mathrm{C}$ with $0 \% \mathrm{CO}_{2}$.

Cell-based degrader assays and western blotting. For assaying degrader activity, cells were seeded (500,000 for $231 \mathrm{MFP}$ cells) into $6 \mathrm{~cm}$ tissue culture plates (Corning) in $2.0-2.5 \mathrm{~mL}$ of media and allowed to adhere overnight. The following morning, media was replaced with complete media containing the desired concentration of CDDO-JQ1 (or related CDDO-based degrader) diluted from a 1,000 $\times$ stock in DMSO. For rescue studies, the cells were pre-treated with proteasome inhibitor (bortezomib, $1 \mu \mathrm{M}$ ), E1 inhibitor (MLN7243, $1 \mu \mathrm{M}$ ) or NEDDylation inhibitor (MLN4924, $1 \mu \mathrm{M}$ ) for $30 \mathrm{~min}$. Cells were subsequently treated with vehicle DMSO or degraders for $12 \mathrm{~h}$. To harvest cells, media was aspirated and cells were washed with $500 \mu \mathrm{L}$ PBS then $100 \mu \mathrm{L}$ Radioimmunoprecipitation assay buffer (RIPA buffer) was added to each well and incubated 5 min on ice before scraping and transferring to Eppendorf tubes. The collected cells were vortexed vigorously in the lysis buffer and allowed to sit on ice for 5 additional min before cellular debris was pelleted by spinning at maximum speed for $10 \mathrm{~min}$ at $4{ }^{\circ} \mathrm{C}$. Supernatant was transferred to new tubes and total protein was normalized by Pierce BCA Protein Assay. Samples were denatured by addition of $4 \times$ Laemmli's Loading dye and $30 \mu \mathrm{g}$ of protein was loaded onto $4-20 \%$ TGX Precast gels (BioRad). After gel electrophoresis, proteins were transferred to a nitrocellulose membrane using semi-dry transfer on a Trans-Blot Turbo (BioRad) over $7 \mathrm{~min}$. The membrane was then incubated for $1 \mathrm{~h}$ in $5 \%$ bovine serum albumin (BSA) in tris-buffered saline containing Tween 20 (TBST) before incubation with the corresponding primary antibody overnight at $4{ }^{\circ} \mathrm{C}$. The membranes were washed in TBST before a $1 \mathrm{~h}$ room temperature incubation with secondary antibodies. After a final set of washes, blots were imaged on a LiCor CLX imager and band intensities were quantified using ImageJ software (Supplementary Information). 
Received: 19 July 2020; Accepted: 27 August 2020

Published online: 23 September 2020

\section{References}

1. Bondeson, D. P. \& Crews, C. M. Targeted protein degradation by small molecules. Annu. Rev. Pharmacol. Toxicol. 57, 107-123 (2017).

2. Burslem, G. M. \& Crews, C. M. Small-molecule modulation of protein homeostasis. Chem. Rev. 117, 11269-11301 (2017).

3. Lai, A. C. \& Crews, C. M. Induced protein degradation: An emerging drug discovery paradigm. Nat. Rev. Drug Discov. 16, 101-114 (2017).

4. Fisher, S. L. \& Phillips, A. J. Targeted protein degradation and the enzymology of degraders. Curr. Opin. Chem. Biol. 44, 47-55 (2018).

5. Churcher, I. Protac-induced protein degradation in drug discovery: Breaking the rules or just making new ones?. J. Med. Chem. 61, 444-452 (2018).

6. Chamberlain, P. P. \& Hamann, L. G. Development of targeted protein degradation therapeutics. Nat. Chem. Biol. 15, 937-944 (2019).

7. Maniaci, C. \& Ciulli, A. Bifunctional chemical probes inducing protein-protein interactions. Curr. Opin. Chem. Biol. 52, 145-156 (2019).

8. Scheepstra, M., Hekking, K. F. W., van Hijfte, L. \& Folmer, R. H. A. Bivalent ligands for protein degradation in drug discovery. Comput. Struct. Biotechnol. J. 17, 160-176 (2019).

9. Verma, R., Mohl, D. \& Deshaies, R. J. harnessing the power of proteolysis for targeted protein inactivation. Mol. Cell 77, 446-460 (2020).

10. Gerry, C. J. \& Schreiber, S. L. Unifying principles of bifunctional, proximity-inducing small molecules. Nat. Chem. Biol. 16, 369-378 (2020).

11. Deshaies, R. J. Multispecific drugs herald a new era of biopharmaceutical innovation. Nature 580, 329-338 (2020).

12. Vassilev, L. T. et al. In vivo activation of the p53 pathway by small-molecule antagonists of MDM2. Science 303, 844-848 (2004).

13. Winter, G. E. et al. Phthalimide conjugation as a strategy for in vivo target protein degradation. Science 348, 1376-1381 (2015).

14. Zengerle, M., Chan, K.-H. \& Ciulli, A. Selective small molecule induced degradation of the BET bromodomain protein BRD4. ACS Chem. Biol. 10, 1770-1777 (2015).

15. Rape, M. Ubiquitylation at the crossroads of development and disease. Nat. Rev. Mol. Cell Biol. 19, 59-70 (2018).

16. Spradlin, J. N. et al. Harnessing the anti-cancer natural product nimbolide for targeted protein degradation. Nat. Chem. Biol. 15, 747-755 (2019).

17. Tong, B. et al. A nimbolde-based kinase degrader preferentially degrades oncogenic BCR-ABL. ACS Chem. Biol. 15, 1788-1794 (2020).

18. Ward, C. C. et al. Covalent ligand screening uncovers a RNF4 E3 ligase recruiter for targeted protein degradation applications. ACS Chem. Biol. 14, 2430-2440 (2019).

19. Zhang, X., Crowley, V. M., Wucherpfennig, T. G., Dix, M. M. \& Cravatt, B. F. Electrophilic PROTACs that degrade nuclear proteins by engaging DCAF16. Nat. Chem. Biol. 15, 737-746 (2019).

20. Serafimova, I. M. et al. Reversible targeting of noncatalytic cysteines with chemically tuned electrophiles. Nat. Chem. Biol. 8, 471-476 (2012).

21. Miller, R. M., Paavilainen, V. O., Krishnan, S., Serafimova, I. M. \& Taunton, J. Electrophilic fragment-based design of reversible covalent kinase inhibitors. J. Am. Chem. Soc. 135, 5298-5301 (2013).

22. Bradshaw, J. M. et al. Prolonged and tunable residence time using reversible covalent kinase inhibitors. Nat. Chem. Biol. 11, 525-531 (2015).

23. Bandyopadhyay, A. \& Gao, J. Targeting biomolecules with reversible covalent chemistry. Curr. Opin. Chem. Biol. 34, 110-116 (2016).

24. Krenske, E. H., Petter, R. C. \& Houk, K. N. Kinetics and thermodynamics of reversible thiol additions to mono- and diactivated michael acceptors: Implications for the design of drugs that bind covalently to cysteines. J. Org. Chem. 81, 11726-11733 (2016).

25. Jackson, P. A., Widen, J. C., Harki, D. A. \& Brummond, K. M. Covalent modifiers: A chemical perspective on the reactivity of a, $\beta$-unsaturated carbonyls with Thiols via hetero-Michael addition reactions. J. Med. Chem. 60, 839-885 (2017).

26. Guo, W.-H., Qi, X., Liu, Y., Chung, C.-I., Bai, F., Lin, X., Lu, D., Wang, L., Chen, J., Nomie, K. J., Li, F., Wang, M. C., Shu, X., Onuchic, J. N., Woyach, J. A., Wang, M. L., Wang, J. (2020) Enhancing intracellular concentration and target engagement of PROTACs with reversible covalent chemistry. bioRxiv 2019.12.30.873588.

27. Gabizon, R. et al. Efficient targeted degradation via reversible and irreversible covalent PROTACs. J. Am. Chem. Soc. 142, 11316 (2020).

28. Yates, M. S. et al. Pharmacodynamic characterization of chemopreventive triterpenoids as exceptionally potent inducers of Nrf2regulated genes. Mol. Cancer Ther. 6, 154-162 (2007).

29. Liby, K. T. \& Sporn, M. B. Synthetic oleanane triterpenoids: Multifunctional drugs with a broad range of applications for prevention and treatment of chronic disease. Pharmacol. Rev. 64, 972-1003 (2012).

30. Sporn, M. B. et al. New synthetic triterpenoids: Potent agents for prevention and treatment of tissue injury caused by inflammatory and oxidative stress. J. Nat. Prod. 74, 537-545 (2011).

31. Lu, J. et al. Hijacking the E3 ubiquitin ligase cereblon to efficiently target BRD4. Chem. Biol. 22, 755-763 (2015).

32. Filippakopoulos, P. et al. Selective inhibition of BET bromodomains. Nature 468, 1067-1073 (2010).

33. Tyler, D. S. et al. Click chemistry enables preclinical evaluation of targeted epigenetic therapies. Science 356, 1397-1401 (2017).

34. Cinelli, M. A., Cordero, B., Dexheimer, T. S., Pommier, Y. \& Cushman, M. Synthesis and biological evaluation of 14-(aminoalkylaminomethyl)aromathecins as topoisomerase I inhibitors: Investigating the hypothesis of shared structure-activity relationships. Bioorg. Med. Chem. 17, 7145-7155 (2009).

35. Taguchi, K. et al. Keapl degradation by autophagy for the maintenance of redox homeostasis. Proc. Natl. Acad. Sci. USA 109, 13561-13566 (2012).

36. Maple, H. J., Clayden, N., Baron, A., Stacey, C. \& Felix, R. Developing degraders: Principles and perspectives on design and chemical space. Med. Chem. Commun. 10, 1755-1764 (2019).

37. Neklesa, T. K. et al. Small-molecule hydrophobic tagging-induced degradation of HaloTag fusion proteins. Nat. Chem. Biol. 7, 538-543 (2011).

38. Tae, H. S. et al. Identification of hydrophobic tags for the degradation of stabilized proteins. ChemBioChem 13, 538-541 (2012).

39. Los, G. V. et al. HaloTag: A novel protein labeling technology for cell imaging and protein analysis. ACS Chem. Biol. 3, 373-382 (2008).

40. Long, M. J. C., Gollapalli, D. R. \& Hedstrom, L. Inhibitor mediated protein degradation. Chem. Biol. 19, 629-637 (2012). 
41. Gustafson, J. L. et al. Small-molecule-mediated degradation of the androgen receptor through hydrophobic tagging. Angew. Chem. Int. Ed. Engl. 54, 9659-9662 (2015).

42. Bhandari, P., Patel, N. K., Gangwal, R. P., Sangamwar, A. T. \& Bhutani, K. K. Oleanolic acid analogs as NO, TNF- $\alpha$ and IL-1 $\beta$ inhibitors: Synthesis, biological evaluation and docking studies. Bioorg. Med. Chem. Lett. 24, 4114-4119 (2014).

43. Honda, T., Finlay, H. J., Gribble, G. W., Suh, N. \& Sporn, M. B. New enone derivatives of oleanolic acid and ursolic acid as inhibitors of nitric oxide production in mouse macrophages. Bioorg. Med. Chem. Lett. 7, 1623-1628 (1997).

44. Lu, M. et al. Discovery of a Keap1-dependent peptide PROTAC to knockdown Tau by ubiquitination-proteasome degradation pathway. Eur. J. Med. Chem. 146, 251-259 (2018).

45. Canning, P., Sorrell, F. J. \& Bullock, A. N. Structural basis of Keap1 interactions with Nrf2. Free Radic. Biol. Med. 88, 101-107 (2015).

46. Canning, P. et al. Structural basis for Cul3 protein assembly with the BTB-Kelch family of E3 ubiquitin ligases. J. Biol. Chem. 288, 7803-7814 (2013).

47. Tran, K. T. et al. A comparative assessment study of known small-molecule Keap1-Nrf2 protein-protein interaction inhibitors: Chemical synthesis, binding properties, and cellular activity. J. Med. Chem. 62, 8028-8052 (2019).

48. Yamamoto, M., Kensler, T. W. \& Motohashi, H. The KEAP1-NRF2 System: A thiol-based sensor-effector apparatus for maintaining redox homeostasis. Physiol. Rev. 98, 1169-1203 (2018).

49. Cleasby, A. et al. Structure of the BTB domain of Keap1 and its interaction with the triterpenoid antagonist CDDO. PLoS ONE 9, e98896 (2014).

50. Huerta, C. et al. Characterization of novel small-molecule NRF2 activators: Structural and biochemical validation of stereospecific KEAP1 binding. Biochem. Biophys. Acta. 1860, 2537-2552 (2016).

51. Saito, R. et al. Characterizations of three major cysteine sensors of Keap1 in stress response. Mol. Cell. Biol. 36, 271-284 (2015).

52. Takaya, K. et al. Validation of the multiple sensor mechanism of the Keap1-Nrf2 system. Free Radical Biol. Med. 53, 817-827 (2012).

53. Wong, M. H. L. et al. Design and synthesis of irreversible analogues of bardoxolone methyl for the identification of pharmacologically relevant targets and interaction sites. J. Med. Chem. 59, 2396-2409 (2016).

54. Ahmad, R., Raina, D., Meyer, C., Kharbanda, S. \& Kufe, D. Triterpenoid CDDO-Me blocks the NF-kappaB pathway by direct inhibition of IKKbeta on Cys-179. J. Biol. Chem. 281, 35764-35769 (2006).

55. Yore, M. M., Kettenbach, A. N., Sporn, M. B., Gerber, S. A. \& Liby, K. T. Proteomic analysis shows synthetic oleanane triterpenoid binds to mTOR. PLoS ONE 6(7), e22862. https://doi.org/10.1371/journal.pone.0022862 (2011).

56. Senkane, K. et al. The proteome-wide potential for reversible covalency at cysteine. Angew. Chem. Int. Ed. Engl. 58, 11385-11389 (2019).

57. Ottis, P. et al. Cellular resistance mechanisms to targeted protein degradation converge toward impairment of the engaged ubiquitin transfer pathway. ACS Chem. Biol. 14, 2215-2223 (2019).

58. Zhang, L., Riley-Gillis, B., Vijay, P. \& Shen, Y. Acquired resistance to BET-PROTACs (proteolysis-targeting chimeras) caused by genomic alterations in core components of E3 ligase complexes. Mol. Cancer Ther. 18, 1302-1311 (2019).

59. Jessani, N. et al. Carcinoma and stromal enzyme activity profiles associated with breast tumor growth in vivo. Proc. Natl. Acad. Sci. USA 101, 13756-13761 (2004).

\section{Acknowledgements}

We thank the members of the Nomura Research Group, the Maimone lab, and the Novartis Institutes for BioMedical Research for critical reading of the manuscript. We are grateful to Dr. Hasan Celik for NMR spectroscopic assistance and NIH Grant S10OD024998. This work was supported by the Novartis Institutes for BioMedical Research, the Novartis-Berkeley Center for Proteomics and Chemistry Technologies, the Mark Foundation for Cancer Research ASPIRE award, and NIH (R01CA240981, F31CA239327).

\section{Author contributions}

T.J.M. and D.K.M. conceived the project, supervised the research, and drafted the manuscript with aid from B.T. and M.L; B.T., M.L., Y.X., and J.N.S. performed the experiments and analyzed the data; J.A.T., J.M.M., and M.S. provided intellectual contributions and insight into project directions; all authors edited the paper.

\section{Competing interests}

J.A.T, J.M.M, M.S. are employees of Novartis Institutes for BioMedical Research. This study was funded by the Novartis Institutes for BioMedical Research and the Novartis-Berkeley Center for Proteomics and Chemistry Technologies. D.K.N. is a co-founder, shareholder, and adviser for Artris Therapeutic and Frontier Medicines. T.J.M., B.T., Y.X., M.L., and J.N.S. declare no competing interests.

\section{Additional information}

Supplementary information is available for this paper at https://doi.org/10.1038/s41598-020-72491-9.

Correspondence and requests for materials should be addressed to T.J.M. or D.K.N.

Reprints and permissions information is available at www.nature.com/reprints.

Publisher's note Springer Nature remains neutral with regard to jurisdictional claims in published maps and institutional affiliations.

Open Access This article is licensed under a Creative Commons Attribution 4.0 International License, which permits use, sharing, adaptation, distribution and reproduction in any medium or
format, as long as you give appropriate credit to the original author(s) and the source, provide a link to the Creative Commons licence, and indicate if changes were made. The images or other third party material in this article are included in the article's Creative Commons licence, unless indicated otherwise in a credit line to the material. If material is not included in the article's Creative Commons licence and your intended use is not permitted by statutory regulation or exceeds the permitted use, you will need to obtain permission directly from the copyright holder. To view a copy of this licence, visit http://creativecommons.org/licenses/by/4.0/.

(C) The Author(s) 2020 